\title{
Mobile Crowdsensing for the Juxtaposition of Realtime Assessments and Retrospective Reporting for Neuropsychiatric Symptoms
}

\author{
Rüdiger Pryss ${ }^{1}$, Thomas Probst ${ }^{1}$, Winfried Schlee ${ }^{2}$, Johannes Schobel ${ }^{1}$, \\ Berthold Langguth ${ }^{2}$, Patrick Neff ${ }^{3}$, Myra Spiliopoulou ${ }^{4}$, Manfred Reichert ${ }^{1}$ \\ ${ }^{1}$ Institute of Databases and Information Systems, Ulm University, Germany \\ ${ }^{2}$ Clinic and Policlinic for Psychiatry and Psychotherapy, University of Regensburg, Germany \\ ${ }^{3}$ Neuroplasticity and Learning in the Healthy Aging Brain, University of Zurich, Switzerland \\ ${ }^{4}$ Department of Technical and Business Information Systems, Otto-von-Guericke-University Magdeburg, Germany \\ ${ }^{1}\{$ ruediger.pryss, thomas.probst, johannes.schobel, manfred.reichert\}@uni-ulm.de \\ winfried.schlee@gmail.com, patrick.neff@uzh.ch,berthold.langguth@medbo.de,myra@iti.cs.uni-magdeburg.de
}

\begin{abstract}
Many symptoms of neuropsychiatric disorders such as tinnitus are subjective and vary over time. Usually, in interviews or self-report questionnaires, patients are asked to report symptoms as well as their severity and duration retrospectively. However, only little is known to what degree such retrospective reports reflect the symptoms experienced in daily life some time ago. Mobile technologies can help to bridge this gap: mobile self-help services allow patients to record their symptoms prospectively when (or shortly after) they occur in daily life. In this study, we present results that we obtained with the mobile crowdsensing platform TrackYourTinnitus to show that there is a discrepancy between the prospective assessment of symptom variability and the retrospective report thereof. To be more precise, we evaluated the real-time entries provided to the platform by individuals experiencing tinnitus. The results indicate that mobile technologies like the TrackYourTinnitus crowdsensing platform may go beyond the role of an assistive service for patients by contributing to more accurate diagnosis and, hence, to a more elaborated treatment.
\end{abstract}

Keywords-Prospective assessment of neuropsychiatric symptoms, retrospective assessment of neuropsychiatric symptoms, self-help mobile applications, tinnitus, mobile data collection, mobile crowdsensing services

\section{INTRODUCTION}

The assessment of neuropsychiatric symptoms is essential in medicine and psychology. For many neuropsychiatric disorders, the duration of the symptoms constitutes an essential factor for diagnosis. Hence, physicians and psychologists need not only reliably assess symptoms and their severity, but also the duration the patient suffers from the symptoms. For example, a major depressive episode can only be diagnosed when the patients suffer from depressive symptoms for at least two weeks. As indicators for most of these symptoms are subjective, the assessment of the symptoms and their duration is typically based on subjective, retrospective reports of the patients. In this context, the question emerges, to what degree the patients are able to remember the severity and duration of the symptoms they actually experienced. Mobile technologies can effectively help to shed light on this question, and also enrich the retrospective reports of the patients with descriptions of the symptoms as they were perceived at the time they occurred. In this work, we describe how the mobile crowdsensing platform TrackYourTinnitus [1]-[3] offers prospective selfreporting facilities to patients. We present findings that aim to discover differences between prospective and retrospective assessments of symptom "tinnitus loudness". Tinnitus can be described as the phantom perception of sound; depending on the definition of tinnitus and its duration, the age, and the birth cohort, between $5.1 \%$ and $42.7 \%$ of the population worldwide experience tinnitus at least once in their lifetime [4]. Tinnitus is variable between patients (inter-individual variability) and also within patients (intraindividual variability). The treatment of tinnitus and the early diagnosis of potential comorbidities require assessments on several symptoms, including loudness and variation of the perceived sound(s), distress caused by tinnitus, impact of tinnitus on sleeping behavior, comorbidities, social activity, concentration, and so forth.

The limited (ecological) validity of retrospective selfreports has been shown in several studies on other neuropsychiatric disorders. For example, [5] assessed physical activities of patients with eating disorders by retrospective self-reports as well as by prospective assessments with an accelerometer. Patients reported significantly less physical activity retrospectively than what was measured prospectively by the accelerometer. The authors of [6], in turn, investigated retrospectively as well as prospectively assessed anxiety and cognition in patients with agoraphobia. While anxiety did not differ between retrospective and prospective assessments, cognition differed.

Such results highlight the potential of Ecological Momentary Assessment (EMA; also known as: ambulatory assessment \& experience sampling) to support clinicians in assessing neuropsychiatric symptoms accurately and in making valid diagnoses. In EMA, the variable in question (e.g., symptoms) is assessed repeatedly in daily life [7]. Instead of retrospectively asking the individuals (through an 
interview or questionnaire) how strongly they experienced a symptom in a given past time interval, the individuals are asked how they currently experience the symptom. In turn, this is done at several time points within the given time interval.

In the aforementioned studies, prospective and retrospective assessments were juxtaposed manually. To exploit effectively the prospective assessments in a clinical setting, an integrated solution is needed, i.e., the EMA of a given patient should be transferred automatically to a database and be made available to the responsible clinician(s), given the consent of the patients. Note that it has been already reported that electronic systems are appreciated by study participants [8], increase data accuracy [9], lead to more complete datasets [10], and reduce costs [11] compared to traditional paper-based methods. However, the exploitation of the prospective assessments next to the retrospective reports has not been investigated in the area of tinnitus yet.

This paper presents the TrackYourTinnitus (TYT) mobile crowdsensing platform [1]-[3] for the juxtaposition of retrospective and prospective assessments of tinnitus-associated neuropsychiatric symptoms. Further, with a focus on tinnitus loudness, we elaborate in what way the prospective data are collected and maintained for further usage.

Related work is discussed in Section II. In Section III, we illustrate the mobile crowdsensing platform of TYT and explain the workflow for the collection and maintenance of prospective assessments. Section IV presents the data and the statistics used for the juxtaposition of the prospective with the retrospective assessments. In Section V, we present the results of the statistical analyses, which suggest that integrating prospective assessments into the diagnostic-therapeutic process is important for optimizing diagnostics and, hence, treatments. Finally, a summary and an outlook is provided in Section VI.

\section{RELATED WORK}

In general, mobile crowdsensing is an emerging research topic in various application domains [12], [13]. Interestingly, in the medical domain, mobile crowdsensing applications have been less proposed so far. The fact that the medical domain is less considered might be related to legal and data privacy issues [14]. However, using mobile crowdsensing in the medical context is promising [15] as mobile crowdsensing has unique features to gather valuable data [16]. In particular, it allows gathering context-aware [17] as well as daily-life data [18] more effectively.

Besides TrackYourTinnitus, two other studies presented EMA approaches to track tinnitus in daily life [19], [20]. Furthermore, EMA approaches capturing other aspects such as pain [21] and feelings [22] in daily life were scientifically evaluated. In addition, EMA approaches were studied in the areas of mood disorders and mood dysregulation [23], [24] as well as in the ones of substance use [25] and eating disorders [26]. In psychotherapy research, EMA has been investigated as predictors of patient progress [27]. Although most neuropsychiatric symptoms are subjective experiences and, thus, most EMA approaches use self-reports to capture these symptoms, some neuropsychiatric symptoms are behavioral (e.g., avoidance in anxiety disorders) or physiological (e.g., increase of heart rate in anxiety disorders) symptoms. Note that mobile systems offer opportunities to measure behavioral or physiological data in daily life [28]. In summary, EMA approaches are considered to offer unprecedented opportunities to study neuropsychiatric symptoms under ecologically valid conditions [29], even though the utilization of its possibilities is still in its infancy, especially in the medical domain.

\section{TRACK YourTinnitus PlatForm}

TrackYourTinnitus (TYT) is a mobile crowdsensing [12] platform, which comprises a website for user registration, two mobile applications (for iOS and Android), and a MySQL database as a central repository for the data collected [2], which can be made available to the clinicians and researchers. The website further provides two other important features: (1) users can visualize recorded tinnitus data and (2) they can report on their current tinnitus treatment. In general, TYT was developed to track the individual tinnitus perception of users. In order to be able to track the daily tinnitus perception, the following procedure must be accomplished by a user (cf. Fig. 1).

First, users have to create a TYT account. The account can be either created through the website or with the use of the mobile applications.

Second, after registering, users have to fill in three registration questionnaires (cf. Fig. 1). First, they have to fill in the "Mini-TQ-12" questionnaire (cf. Fig. 1, MiniTQ-12 [30]), which measures tinnitus-related psychological problems. Second, they have to fill in the "Tinnitus Sample Case History Questionnaire (TSCHQ)" (cf. Fig. 1, TSCHQ [31]). The latter determines the current tinnitus status of the user as well as his tinnitus history. Note that the TSCHQ questionnaire comprises the question being important for the results of this paper. To be more precise, the 11th item of the TSCHQ questionnaire [31] asks the user retrospectively whether or not the tinnitus loudness varies from day to day. Finally, users have to fill in the "Worst Symptom" questionnaire (cf. Fig. 1, Worst Symptom Questionnaire), which asks the users about the worst symptom currently caused by their tinnitus. While the first two questionnaires constitute already used instruments, the third one was newly developed. Altogether, the completion of the three questionnaires with their 58 questions in total constitutes a prerequisite to be able to use the TYT website features as well as the mobile applications.

Third, after completing the registration questionnaires, the user can use the mobile applications to track the daily 


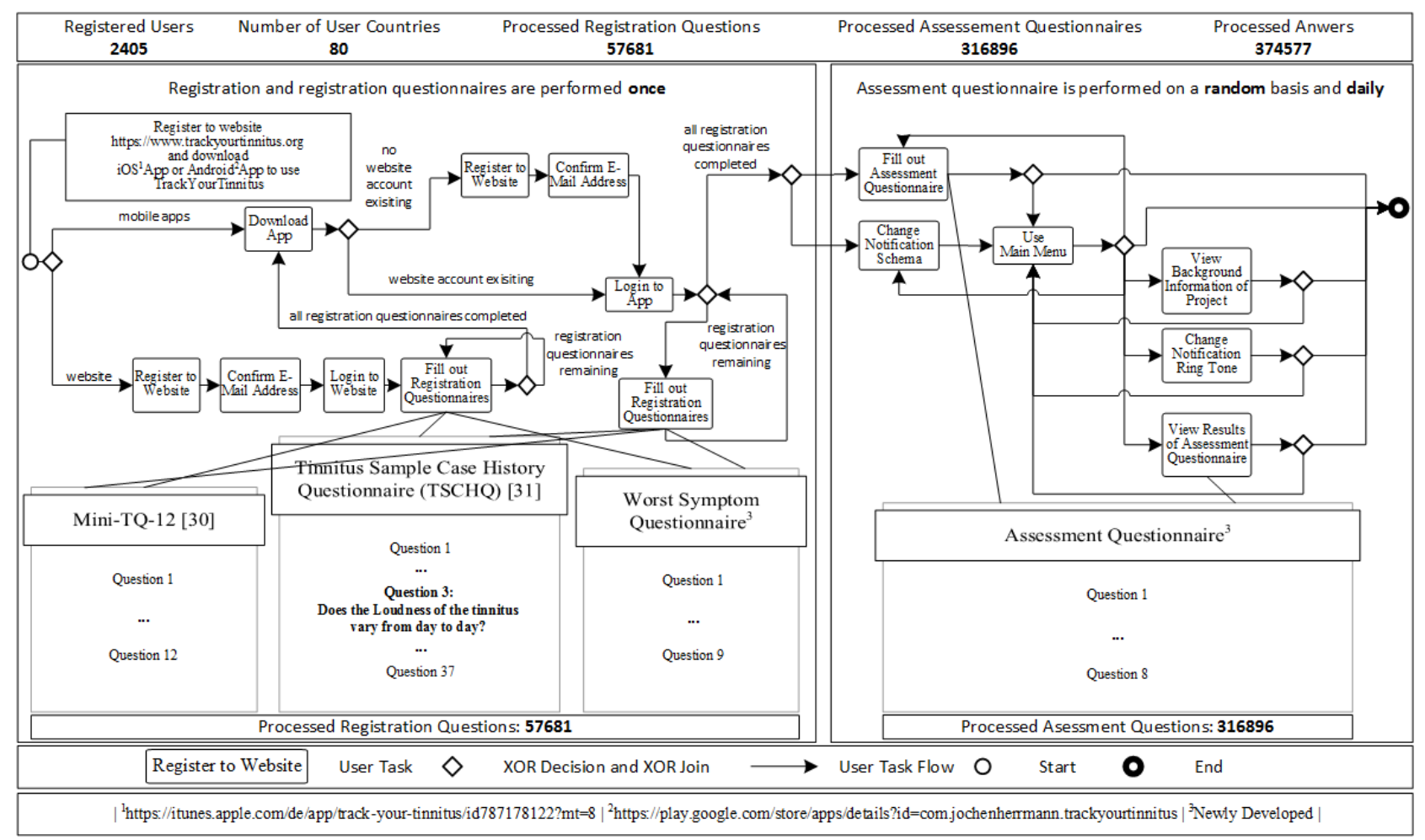

Figure 1: TrackYourTinnitus Crowdsensing Platform

tinnitus perception. For this purpose, the user has to log in to the Android or iOS mobile application. Then, they are asked to fill in the assessment questionnaire developed for TYT (cf. Fig. 1, Assessment Questionnaire). This questionnaire comprises 8 questions and rates the tinnitus perception of the user (e.g., current tinnitus loudness).

Fourth, the assessment questionnaire is provided in two ways: (1) the mobile application automatically provides the questionnaire to the users or (2) they make the conscious decision to fill in the questionnaire. The first way is the desired procedure, which is realized as follows: The assessment questionnaire is randomly presented to the user up to 12 times per day (i.e., users can define an individual time schedule). Therefore, notification features for Android and iOS as well as a notification algorithm were realized [2]. This procedure ensures that (1) users cannot foresee the time when being asked and (2) users are asked in various daily situations. This randomized approach was realized to improve the ecological validity of the applied method.

Fifth, while filling in the assessment questionnaire, the smart mobile device of a user records the environmental sound level.

Finally, results gathered with the assessment questionnaire and sound recording are transferred to the TYT database. The latter, in turn, offers features enabling researchers to evaluate gathered user data. Note that this feature was used for obtaining the presented results.
The TYT crowdsensing platform is currently provided in English, German, and Dutch. The current number (February 2017) of processed questionnaires and registered users can be obtained from Fig. 1. As can be seen, the TYT mobile crowdsensing platform is frequently used. So far, users from 80 countries have provided data to the platform. It is noteworthy that users from seven countries provide the major part of all gathered data. To be more precise, $35.4 \%$ users come from Germany, $14.6 \%$ from the US, $5.8 \%$ from the Netherlands, $5.8 \%$ from the UK, $3.7 \%$ from Switzerland, $3.3 \%$ from Canada, and 2.9\% from France. Further note that analyzing data gathered with mobile crowdsensing techniques might reveal new valuable insights to tinnitus [32] in particular and other chronic diseases in general.

\section{DATA AND STATISTICS}

The current analysis relies on an export of the TYT database made in February 2017. ${ }^{1}$ In the current study, we analyzed two variables. The first one assessed the variability of tinnitus retrospectively. The following question, which was asked once during the TYT registration process, was used to measure retrospectively whether or not the tinnitus loudness varies: "Does the LOUDNESS of the tinnitus vary from day to day?" with the options to respond with "yes" or "no". The second variable measured tinnitus loudness

\footnotetext{
${ }^{1}$ That means, the 1291 users coming from the 80 countries provided meaningful data sets for the presented analysis
} 
prospectively. Thereby, the users rated at several points in time during their daily life the following question "How LOUD is the tinnitus right now?" with a slider ranging from 0 (i.e., not audible) to 1 (i.e., maximal loudness). To be able to compare the retrospective assessments on day-to-day variation with the variation of the prospective assessments, we had to focus our analysis on the day-today variation (i.e., excluding within-day variation) of the prospective assessments. Therefore, for each patient with more than one prospective tinnitus loudness assessment per day, we calculated the mean of their within-day prospective assessments in order to have one prospective loudness assessment per day.

Then, the variability of these prospective loudness ratings was calculated for each user as the standard deviation (SD) of all their prospective loudness ratings (on a day-to-day level). To obtain a meaningful day-to-day variability of the prospective tinnitus loudness ratings, only those users who provided a prospective tinnitus loudness assessment at least at 10 days were investigated. We used a t-test for independent samples (two-tailed, with $p<0.05$ indicating a statistically significant result) to evaluate whether the SD of the prospective tinnitus loudness assessments belonging to the users retrospectively describing their tinnitus loudness as non-varying significantly differs from the SD of the prospective tinnitus loudness assessments belonging to the users retrospectively describing their tinnitus loudness as varying. After applying these criteria, plus the exclusion of assessments with an inter-assessment interval of 15 mins or less [2], and the exclusion of a study sample recruited differently (e.g., for specific studies) than the rest of the TYT users, $N=260$ users remained for the statistical analysis. Of these $N=260$ users, $n=47(18 \%)$ reported retrospectively that the tinnitus loudness does not vary from day to day and $n=213(82 \%)$ reported retrospectively that the tinnitus loudness varies from day to day.

While the users retrospectively rating the tinnitus loudness as stable provided prospective assessments of tinnitus loudness for $M=37.57(S E=4.83)$ days (in an average interval of $M=3.29$ months), the users retrospectively rating the tinnitus as varying provided data for $M=39.81$ $(S E=3.65)$ days (in an average interval of $M=4.35$ months). We also ran the statistical test for users with prospective loudness assessments for at least 25 days in order to investigate whether the results can be replicated. After applying these stricter inclusion criteria, $N=128$ users were remaining for the statistical analysis. Of these users, $n=25$ (20\%) reported retrospectively that the tinnitus loudness does not vary from day to day, whereas $n=103$ $(80 \%)$ reported retrospectively that the tinnitus loudness varies from day to day. Here, the users retrospectively rating the tinnitus loudness as stable provided prospective assessments of tinnitus loudness for $M=56.60(S E=7.17)$ days (average time interval the assessments were provided in: $M=3.76$ months), whereas the users retrospectively rating the tinnitus as varying provided data for $M=66.16$ $(S E=6.63)$ days (average time interval the assessments were provided in: $M=4.87$ months). In order to investigate whether the TYT users retrospectively rating the tinnitus loudness as non-varying differ in baseline variables from users retrospectively rating the tinnitus loudness as varying, independent t-tests and chi-squared tests were performed (again two-tailed with $p<0.05$ as statistical significance level).

\section{RESUlts}

The comparisons between the users retrospectively rating the tinnitus as not varying and the users retrospectively rating the tinnitus as varying are given in Table I. It can be seen that TYT users who retrospectively rated their tinnitus loudness as non-varying from day to day did not significantly differ in the average variation (SD) of the prospective tinnitus loudness assessments from the TYT users who retrospectively rated their tinnitus loudness as varying from day to day. This result emerged when TYT users providing prospective tinnitus loudness assessments at least at 10 days were analyzed as well as when TYT users providing prospective tinnitus loudness assessments at least at 25 days were analyzed. Moreover, no significant differences in baseline variables (i.e., gender, age, and tinnitus duration) were found between users with retrospectively varying and users with retrospectively non-varying tinnitus loudness. However, in the sample consisting of TYT users with at least 10 prospective tinnitus loudness assessments, male users (compared to female users) tended to retrospectively rate the tinnitus loudness more frequently as nonvarying ( $p=0.056)$ and the Mini-TQ-12 [30] score tended to be higher for users who retrospectively rated the tinnitus loudness as varying ( $p=0.089)$. In summary, prospective assessments offer information not covered by retrospective ratings. This makes prospective assessments valuable for diagnostics and treatments.

\section{Summary AND OUTLOOK}

The present study used data from a mobile crowdsensing platform developed for tracking tinnitus in daily life. Retrospective and prospective measurements of variations of tinnitus loudness were compared against each other. We found out that the prospectively measured variation of tinnitus loudness is not significantly different between individuals who retrospectively rated their tinnitus loudness as non-varying and individuals who retrospectively rated their tinnitus loudness as varying. This result is in line with other studies showing that retrospective self-reports differ from prospective assessments. Note that retrospective assessments might be biased (i.e., recall bias). Another reason in the context of our study could be that the individuals who retrospectively rated their tinnitus as non-varying have 
not understood the ups and downs of their tinnitus yet. In psychotherapies with patients suffering from neuropsychiatric symptoms, it is often a first technique to assign the homework to observe the symptoms in order to become aware that the symptoms fluctuate and to learn which factors influence the fluctuations. Possibly, individuals who

\begin{tabular}{|c|c|c|c|}
\hline & $\begin{array}{l}\text { Retrospective } \\
\text { rating of } \\
\text { tinnitus } \\
\text { loudness } \\
\text { variation = no }\end{array}$ & $\begin{array}{l}\text { Retrospective } \\
\text { rating of } \\
\text { tinnitus } \\
\text { loudness } \\
\text { variation = yes }\end{array}$ & Statistics \\
\hline $\begin{array}{c}\text { Male gender } \mathrm{n} \\
(\%)\end{array}$ & $39(84.8)$ & $150(71.1)$ & $\begin{array}{l}\chi^{2}(1)= \\
3.639 \\
p=0.056\end{array}$ \\
\hline $\begin{array}{r}\text { Age } M \\
\text { (SE) }\end{array}$ & $\begin{array}{l}45.6618 \\
(1.8804)\end{array}$ & $\begin{array}{l}47.4519 \\
(0.7928)\end{array}$ & $\begin{array}{c}t(235)= \\
-0.925 \\
p=0.356\end{array}$ \\
\hline $\begin{array}{c}\text { Tinnitus } \\
\text { duration } \\
\text { (subjective } \\
\text { report in years) } \\
\text { M (SE) }\end{array}$ & $\begin{array}{l}13.6569 \\
(2.1066)\end{array}$ & $\begin{array}{l}11.4626 \\
(0.8217)\end{array}$ & $\begin{array}{c}t(59.438)= \\
0.970 \\
p=0.336\end{array}$ \\
\hline $\begin{array}{l}\text { Mini-TQ-12 } \\
\text { [30] score M } \\
\text { (SE) }\end{array}$ & $\begin{array}{l}11.9333 \\
(0.7865)\end{array}$ & $\begin{array}{l}13.5143 \\
(0.3942)\end{array}$ & $\begin{array}{c}t(253)= \\
-1.707 \\
p=0.089\end{array}$ \\
\hline $\begin{array}{l}\text { Variation (SD) } \\
\text { of the } \\
\text { prospective } \\
\text { tinnitus } \\
\text { loudness } \\
\text { assessments M } \\
\text { (SE) }\end{array}$ & $\begin{array}{l}0.148 \\
(0.010)\end{array}$ & $\begin{array}{l}0.145 \\
(0.005)\end{array}$ & $\begin{array}{c}t(258)= \\
0.194 ; \\
p=0.846\end{array}$ \\
\hline $\begin{array}{l}\text { Male gender } \mathrm{n} \\
(\%)\end{array}$ & $21(84.0)$ & $74(72.5)$ & $\begin{array}{c}\chi^{2}(1)= \\
1.397 \\
p=0.237\end{array}$ \\
\hline $\begin{array}{r}\text { Age } M \\
\text { (SE) }\end{array}$ & $\begin{array}{l}47.042 \\
(2.430)\end{array}$ & $\begin{array}{l}49.439 \\
(1.047)\end{array}$ & $\begin{array}{c}t(115)= \\
-0.976 \\
p=0.331\end{array}$ \\
\hline $\begin{array}{c}\text { Tinnitus } \\
\text { duration } \\
\text { (subjective } \\
\text { report in years) } \\
\text { M (SE) }\end{array}$ & $\begin{array}{l}16.563 \\
(3.158)\end{array}$ & $\begin{array}{l}12.215 \\
(1.209)\end{array}$ & $\begin{array}{c}t(31.393)= \\
1.286 ; \\
p=0.208\end{array}$ \\
\hline $\begin{array}{l}\text { Mini-TQ-12 } \\
\text { [30] score M } \\
\text { (SE) }\end{array}$ & $\begin{array}{l}12.560 \\
(1.033)\end{array}$ & $\begin{array}{l}13.109 \\
(0.525)\end{array}$ & $\begin{array}{c}t(124)= \\
-0.467 ; \\
p=0.641\end{array}$ \\
\hline $\begin{array}{l}\text { Variation (SD) } \\
\text { of the } \\
\text { prospective } \\
\text { tinnitus } \\
\text { loudness } \\
\text { assessments M } \\
\text { (SE) }\end{array}$ & $\begin{array}{l}0.143 \\
(0.014)\end{array}$ & $\begin{array}{l}0.130 \\
(0.006)\end{array}$ & $\begin{array}{c}t(126)= \\
0.960 ; \\
p=0.339\end{array}$ \\
\hline \multicolumn{4}{|c|}{$\begin{array}{l}\text { Results of the comparisons between TYT users retrospectively rating the } \\
\text { tinnitus loudness as non-varying and TYT users retrospectively rating the } \\
\text { tinnitus loudness as varying. Note: TYT = TrackYourTinnitus; } M=\text { Mean, } \\
\text { SD = Standard Deviation; Mini-TQ = Tinnitus Questionnaire Short Form }\end{array}$} \\
\hline
\end{tabular}

Table I: Results retrospectively rate symptoms as varying are more trained in self-observing strategies. It could be speculated in this context that longer tinnitus duration increases self-observing and that individuals with longer tinnitus duration rate the tinnitus as non-varying more frequently. Yet, our results have shown that the tinnitus duration did not significantly differ between TYT users retrospectively rating the tinnitus as nonvarying and TYT users retrospectively rating the tinnitus as varying. Nevertheless, individuals who retrospectively assess the symptom as non-varying might gain a deeper understanding of the symptom when receiving feedback on the information that the symptom is prospectively varying. In future studies, we plan to investigate whether it is helpful for patients with tinnitus to receive feedback on tinnitus fluctuations and on correlates of the fluctuations as stored in the TYT platform.

Moreover, a database of EMA assessments could be used to feed the patients' experiences of neuropsychiatric symptoms in daily life back to the responsible clinician(s) (in case the patient agrees with this procedure). This might be helpful to improve the treatment [33] or to support the clinicians in making diagnoses or case conceptualizations. The diagnostic procedure of neuropsychiatric symptoms is usually time- and cost-intensive. Time and costs, in turn, might be saved when patients use mobile systems in daily life to assess neuropsychiatric symptoms prospectively before the appointment for the diagnostic procedure takes place: clinicians, who receive feedback on these assessments, could then adjust the diagnostic procedures to the data and would not have to evaluate the patients' neuropsychiatric symptoms retrospectively. In summary, mobile crowdsensing systems capturing neuropsychiatric symptoms prospectively in daily life appear to offer several advantages that warrant further investigation and major breakthroughs in medical research.

\section{REFERENCES}

[1] R. Pryss, M. Reichert, J. Herrmann, B. Langguth, and W. Schlee, "Mobile Crowd Sensing in Clinical and Psychological Trials - A Case Study," in 28th IEEE Int'l Symposium on Computer-Based Medical Systems. IEEE Computer Society Press, June 2015.

[2] R. Pryss, M. Reichert, B. Langguth, and W. Schlee, "Mobile Crowd Sensing Services for Tinnitus Assessment, Therapy and Research," in IEEE 4th Int'l Conf on Mobile Services. IEEE Computer Society Press, 2015.

[3] W. Schlee, R. Pryss, T. Probst, J. Schobel, A. Bachmeier, M. Reichert, and B. Langguth, "Measuring the moment-tomoment variability of tinnitus: the TrackyourTinnitus smart phone app," Frontiers in Aging Neuroscience, vol. 8, 2016.

[4] A. McCormack, M. Edmondson-Jones, S. Somerset, and D. Hall, "A systematic review of the reporting of tinnitus prevalence and severity," Hearing research, vol. 337, pp. 7079, 2016. 
[5] S. Bratland-Sanda et al., "i'm not physically active-i only go for walks: Physical activity in patients with longstanding eating disorders," International Journal of Eating Disorders, vol. 43, no. 1, pp. 88-92, 2010.

[6] M. Marks and D. Hemsley, "Retrospective versus prospective self-rating of anxiety symptoms and cognitions," Journal of anxiety disorders, vol. 13, no. 5, pp. 463-472, 1999.

[7] T. Trull and U. Ebner-Priemer, "Ambulatory assessment," Annual review of clinical psychology, vol. 9, pp. 151-176, 2013.

[8] S. Lane, N. Heddle, E. Arnold, and I. Walker, "A review of randomized controlled trials comparing the effectiveness of hand held computers with paper methods for data collection," BMC medical informatics and decision making, vol. 6, no. 1, p. 23, 2006.

[9] T. Palermo, D. Valenzuela, and P. Stork, "A randomized trial of electronic versus paper pain diaries in children: impact on compliance, accuracy, and acceptability," Pain, vol. 107, no. 3, pp. 213-219, 2004.

[10] J. Marcano Belisario et al., "Comparison of self-administered survey questionnaire responses collected using mobile apps versus other methods," The Cochrane Library, 2015.

[11] I. Pavlović, T. Kern, and D. Miklavčič, "Comparison of paperbased and electronic data collection process in clinical trials: costs simulation study," Contemporary clinical trials, vol. 30, no. 4, pp. 300-316, 2009.

[12] L. Shu, Y. Chen, Z. Huo, N. Bergmann, and L. Wang, "When Mobile Crowd Sensing Meets Traditional Industry," IEEE Access, 2017.

[13] H. Li, T. Li, and Y. Wang, "Dynamic participant recruitment of mobile crowd sensing for heterogeneous sensing tasks," in 12th International Conference on Mobile Ad Hoc and Sensor Systems. IEEE, 2015, pp. 136-144.

[14] D. Christin, A. Reinhardt, S. Kanhere, and M. Hollick, "A survey on privacy in mobile participatory sensing applications," Journal of Systems and Software, vol. 84, no. 11, pp. 1928-1946, 2011.

[15] R. Ganti, F. Ye, and H. Lei, "Mobile crowdsensing: current state and future challenges," IEEE Communications Magazine, vol. 49, no. 11, 2011.

[16] M. Demirbas et al., "Crowd-sourced sensing and collaboration using twitter," in International Symposium on a World of Wireless Mobile and Multimedia Networks. IEEE, 2010, pp. $1-9$.

[17] H. Ma, D. Zhao, and P. Yuan, "Opportunities in mobile crowd sensing," IEEE Communications Magazine, vol. 52, no. 8, pp. 29-35, 2014.

[18] T. Probst, R. Pryss, B. Langguth, and W. Schlee, "Emotional states as mediators between tinnitus loudness and tinnitus distress in daily life: Results from the "TrackyourTinnitus" application," Scientific reports, vol. 6, 2016.
[19] M. Wilson et al., "Ecological momentary assessment of tinnitus using smartphone technology a pilot study," Otolaryngology-Head and Neck Surgery, p. $0194599815569692,2015$.

[20] J. Henry et al., "Pilot study to evaluate ecological momentary assessment of tinnitus," Ear and hearing, vol. 32, no. 2, p. 179, 2012.

[21] R. Jamison et al., "Electronic diaries for monitoring chronic pain: 1-year validation study," Pain, vol. 91, no. 3, pp. 277285, 2001.

[22] M. Killingsworth and D. Gilbert, "A wandering mind is an unhappy mind," Science, vol. 330, no. 6006, pp. 932-932, 2010 .

[23] S. Wenze and I. Miller, "Use of ecological momentary assessment in mood disorders research," Clinical psychology review, vol. 30, no. 6, pp. 794-804, 2010.

[24] P. Santangelo, M. Bohus, and U. Ebner-Priemer, "Ecological momentary assessment in borderline personality disorder: a review of recent findings and methodological challenges," Journal of Personality Disorders, vol. 28, no. 4, pp. 555-576, 2014.

[25] S. Shiffman, "Ecological momentary assessment (EMA) in studies of substance use." Psychological assessment, vol. 21, no. 4, p. 486, 2009.

[26] S. Engel et al., "Ecological momentary assessment in eating disorder and obesity research: a review of the recent literature," Current psychiatry reports, vol. 18, no. 4, pp. 1-9, 2016.

[27] K. Husen et al., "Daily affect dynamics predict early response in CBT: Feasibility and predictive validity of EMA for outpatient psychotherapy," Journal of Affective Disorders, vol. 206, pp. 305-314, 2016.

[28] U. Ebner-Priemer and T. Kubiak, "Psychological and psychophysiological ambulatory monitoring," European Journal of Psychological Assessment, vol. 23, no. 4, pp. 214-226, 2007.

[29] I. Myin-Germeys et al., "Experience sampling research in psychopathology: opening the black box of daily life," $P s y$ chological medicine, vol. 39, no. 9, p. 1533, 2009.

[30] W. Hiller and G. Goebel, "Rapid assessment of tinnitusrelated psychological distress using the Mini-TQ," Int $J$ Audiol, vol. 43, no. 10, pp. 600-604, 2004.

[31] B. Langguth et al., "Consensus for tinnitus patient assessment and treatment outcome measurement: Tinnitus Research Initiative meeting, Regensburg, July 2006," Progress in brain research, vol. 166, pp. 525-536, 2007.

[32] T. Probst, R. Pryss, B. Langguth, and W. Schlee, "Emotion dynamics and tinnitus: Daily life data from the "TrackyourTinnitus" application," Scientific reports, vol. 6, 2016.

[33] I. Kramer et al., "A therapeutic application of the experience sampling method in the treatment of depression: a randomized controlled trial," World Psychiatry, vol. 13, no. 1, pp. 68-77, 2014. 